\title{
Bioactivity of Ruta graveolens and Satureja montana Essential Oils on Solanum tuberosum Hairy Roots and Solanum tuberosum Hairy Roots with Meloidogyne chitwoodi Co-cultures
}

\author{
Jorge M. S. Faria, ${ }^{\dagger}$ Ana M. Rodrigues, ${ }^{\dagger}$ Inês Sena, ${ }^{\dagger}$ Cristina Moiteiro, ${ }^{\ddagger}$ Richard N. Bennett, ${ }^{\S}$
} Manuel Mota, $\|, \perp$ and A. Cristina Figueiredo ${ }^{*}, \dagger$

${ }^{\dagger}$ Centro de Estudos do Ambiente e do Mar Lisboa, Faculdade de Ciências, Universidade de Lisboa, CBV, C2, Piso 1, Campo Grande, 1749-016 Lisboa, Portugal

† Centro de Química e Bioquímica, Faculdade de Ciências, Universidade de Lisboa, Campo Grande, 1749-016, Lisboa, Portugal

${ }^{\S}$ Universidade de Trás-os-Montes e Alto Douro, Quinta dos Prados, Apartado 1013, 5000-911 Vila Real, Portugal

"NemaLab, ICAAM - Instituto de Ciências Agrárias e Ambientais Mediterrânicas, Universidade de Évora, Núcleo da Mitra, Apartado 94, 7002-554 Évora, Portugal

${ }^{\perp}$ Departamento de Ciências da Vida, Universidade Lusófona de Humanidades e Tecnologias, EPCV, Campo Grande 376, 1749-024 Lisboa, Portugal

\begin{abstract}
As a nematotoxics screening biotechnological system, Solanum tuberosum hairy roots (StHR) and S. tuberosum hairy roots with Meloidogyne chitwoodi co-cultures (StHR/CRKN) were evaluated, with and without the addition of the essential oils (EOs) of Satureja montana and Ruta graveolens. EOs nematotoxic and phytotoxic effects were followed weekly by evaluating nematode population density in the co-cultures as well as growth and volatile profiles of both in vitro cultures types. Growth, measured by the dissimilation method and by fresh and dry weight determination, was inhibited after EO addition. Nematode population increased in control cultures, while in EO-added cultures numbers were kept stable. In addition to each of the EOs main components, and in vitro cultures constitutive volatiles, new volatiles were detected by gas chromatography and gas chromatography coupled to mass spectrometry in both culture types. StHR with CRKN co-cultures showed to be suitable for preliminary assessment of nematotoxic EOs.
\end{abstract}

KEYWORDS: biotransformation, Columbia root-knot nematode, in vitro co-cultures, rue, winter savory, 8-phenyl-2-octanol

\section{INTRODUCTION}

Root-knot nematodes (RKNs), Meloidogyne spp., have been recently ranked first in the top 10 list of plant-parasitic nematodes with scientific and economic importance. ${ }^{1}$ Rootknot is one of the five most damaging potato (Solanum tuberosum L.) pests in modern agriculture. Commonly used nematicides are broad-spectrum synthetic chemicals which have been shown to be linked to environment pollution and undesirable influences on nontarget organisms and human health. ${ }^{2,3}$ This led to the search for environmentally friendly natural nematicides that are, at the same time, cost-effective.

For displaying multiple biological activities, essential oils (EOs) are desirable biopesticides, ${ }^{4}$ able to control not only the targeted pest but also opportunistic species and resistant strains. EOs are complex mixtures of volatiles, mainly products from the plant's secondary metabolism, comprising terpenes (mostly mono-, sesqui-, and a few diterpenes) and phenolic compounds (such as phenylpropanoids), although other groups of compounds can also occur in relevant amounts.

EO nematotoxic activity evaluation is commonly performed by direct contact bioassays and/or greenhouse and field assays. $^{5-9}$ Despite the importance of these tests, the main problems associated with direct contact assays are the fact that they neither assess the phytotoxicity to nor the biotransformation capacity of the host. On the other hand, greenhouse and field assays are very laborious and often environmentally dependent.

As a laboratory model, in vitro co-cultures, that is, the growth of more than one organism or cell type in a combined culture, provide a controlled environment and allow the analysis of metabolomic relationships between plant and nematode at various levels. ${ }^{10,11}$ Particularly important is to follow, simultaneously, if the nematotoxic maintains its activity against the pathogen while not showing phytotoxicity to the host.

Previous work has shown the antihatching potential of Ruta graveolens L. (rue) and Satureja montana L. (winter savory) EOs, and EO hydrocarbon and oxygen-containing molecules fractions, against Columbia root-knot nematode (CRKN, Meloidogyne chitwoodi) hatching in direct contact bioassays. ${ }^{12}$ These EOs have shown diverse behaviors when added to Pinus pinaster shoot cultures and $P$. pinaster with Bursaphelenchus xylophilus in vitro co-cultures. ${ }^{13}$

Using previously established $S$. tuberosum hairy roots (StHR) and $S$. tuberosum hairy roots with $M$. chitwoodi co-cultures $(\mathrm{StHR} / \mathrm{CRKN}),{ }^{10}$ the present work aimed at assessing $R$.

Received: July 22, 2016

Revised: September 14, 2016

Accepted: September 21, 2016

Published: September 21, 2016 

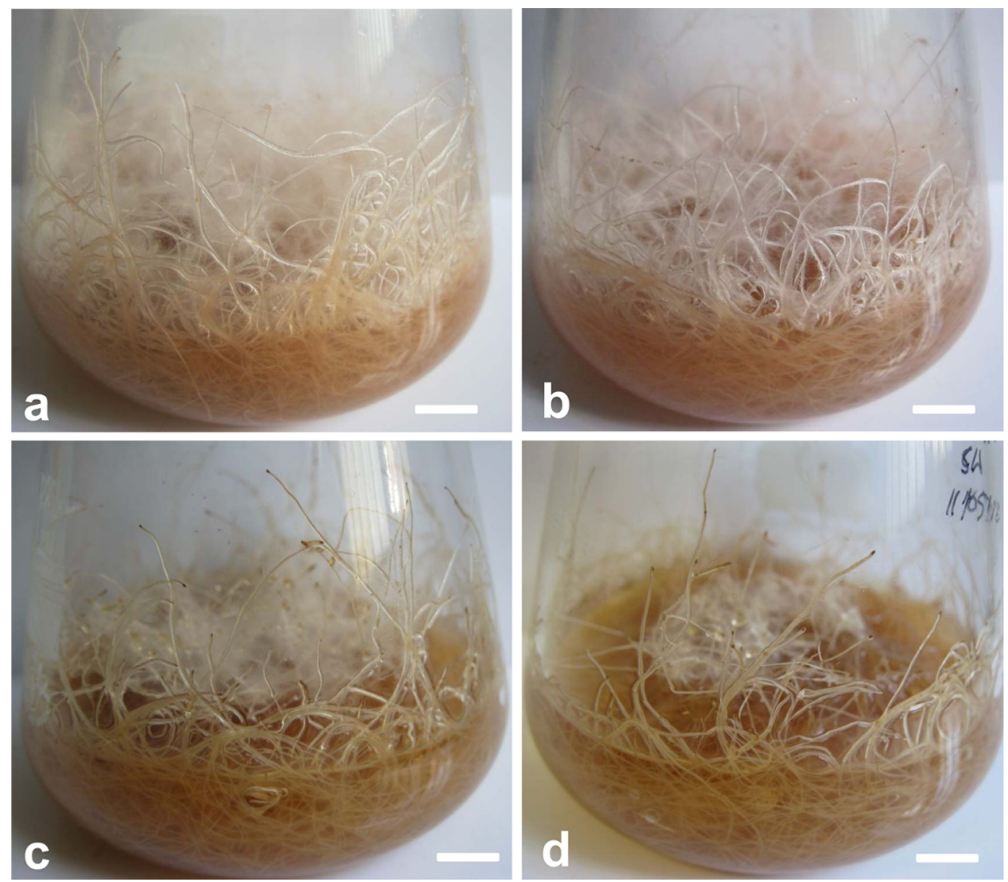

Figure 1. Aspect of (a) Solanum tuberosum hairy roots (StHR), (b) S. tuberosum HR with Meloidogyne chitwoodi co-cultures (StHR/CRKN) and StHR to which (c) Satureja montana and (d) Ruta graveolens EOs were added at $0.5 \mu \mathrm{L} / \mathrm{mL}$, with 5 weeks in culture (1 week after EO addition). Both StHR cultures and StHR/CRKN co-cultures showed similar aspect when grown in EOs-added culture media. Scale bar $1 \mathrm{~cm}$.

graveolens and S. montana EOs nematotoxicity and phytotoxicity by evaluating nematode population density in the cocultures medium as well as growth and volatile profiles of both in vitro culture types.

\section{MATERIALS AND METHODS}

Solanum tuberosum Hairy Roots and S. tuberosum Hairy Roots with Meloidogyne chitwoodi Co-cultures. S. tuberosum hairy roots (StHR) and S. tuberosum HR with Columbia root-knot nematode (CRKN, Meloidogyne chitwoodi) co-cultures (StHR/CRKN) were previously established and routinely maintained in Schenk and Hildebrandt (SH) medium ${ }^{14}$ with $30 \mathrm{~g} / \mathrm{L}$ sucrose in darkness at $24{ }^{\circ} \mathrm{C}$ on orbital shakers at $80 \mathrm{rpm}$, as detailed in Faria et al. ${ }^{10}$

Ruta graveolens and Satureja montana Essential Oils. $R$. graveolens and S. montana essential oils (EOs) were isolated by hydrodistillation from the dried aerial parts sold in local herbal shops as detailed in Faria et al. ${ }^{15}$

Ruta graveolens and Satureja montana Essential Oils Bioactivity Assays. $R$. graveolens and $S$. montana EOs addition phytotoxic effect on StHR and phyto- and nematotoxic effects on StHR/CRKN co-cultures were followed by measuring in vitro cultures growth, nematode population density, and volatiles production during the 7 weeks. To attain this, Erlenmeyer flasks with $100 \mathrm{~mL}$ of SH medium were aseptically inoculated with $1 \mathrm{~g}$ (fresh weight) of StHR or StHR/CRKN co-cultures and maintained as described above. Four weeks following subculture, a 1:1 solution ( $\mathrm{v} / \mathrm{v})$ of $S$. montana or $R$. graveolens EO in methanol (Panreac Química S.A.U., Barcelona, Spain) was added to each culture flask to obtain a final concentration of 0.5 $\mu \mathrm{L} \mathrm{EO} / \mathrm{mL}$ of culture medium. Methanol was chosen due to its high polarity and high solvent capacity. Two types of control cultures, StHR and StHR/CRKN co-cultures without EO, were maintained simultaneously and were processed as the ones to which EOs were added. EO evaporation control experiments were performed by adding the same amount of $\mathrm{EO}$ to flasks containing only basal culture medium and keeping them in the same conditions as the culture flasks throughout the experiment. Two independent experiments were separately run for each EO, and two replicates of each flask were used in each experiment. The data shown were calculated as mean values of all experiments. All statistical analyses were performed using Microsoft Excel 2013.

StHR and StHR/CRKN Growth. StHR and StHR/CRKN growth, in control and EO added cultures, was assessed by the dissimilation method and by fresh and dry weight determination, as detailed in Faria et al. ${ }^{10}$

CRKN Population in Co-culture Medium. CRKN population density (J2 and males) in the liquid culture medium was evaluated by sampling $100 \mu \mathrm{L}$ aliquots of each culture flask at each time-point. Three replicates of each flask were used for counts. Number of dead and live nematodes was recorded using an inverted microscope [Diaphot, Nikon, Japan (40x)].

Essential Oils and Volatiles Chemical Characterization. $R$ graveolens and $S$. montana essential oils chemical profiling was previously detailed in Faria et al. ${ }^{15}$ StHR and StHR/CRKN volatiles were isolated and characterized as described in Faria et al. ${ }^{10}$

Synthesis and Identification of 8-Phenyl-2-octanol. The isolation and characterization of $R$ graveolens EO main constituents permitted the unequivocal identification of 8-phenyl-2-octanone as a dominant ketone ${ }^{13}$ not usually identified in GC-MS analysis of EOs from this species. The occurrence of a new compound in considerable amounts after $R$. graveolens $\mathrm{EO}$ addition to in vitro cultures and following the comparison of its mass spectra to the ones obtained from 8-phenyl-2-octanone suggested the presence of its corresponding alcohol. To obtain high amounts of the unidentified alcohol, the ketone was isolated from $R$. graveolens EO using a methodology adapted from Faria et al. ${ }^{13}$ Purification was performed by fractionation of approximately $1500 \mu \mathrm{L}(1.26 \mathrm{~g})$ of rue $\mathrm{EO}$ on a silica gel column ${ }^{16}$ by elution with $n$-hexane followed by $n$-hexane:ethyl acetate (99.5:0.5 and 99:1). The pure fraction $(153 \mathrm{mg})$, obtained as an oil, was reduced with $\mathrm{NaBH}_{4}(1: 1.2 \mathrm{w} / \mathrm{w})$ to synthesize the respective alcohol, 8phenyl-2-octanol, that was characterized and identified by NMR spectroscopy and GC-MS spectrometry. 1D NMR $\left({ }^{1} \mathrm{H},{ }^{13} \mathrm{C}\right.$, and APT) and 2D NMR (HSQC, HMBC, and COSY) spectra were recorded on Bruker spectrometer CXP400 operating at $400.13 \mathrm{MHz}\left({ }^{1} \mathrm{H}\right)$ and $100.61 \mathrm{MHz}\left({ }^{13} \mathrm{C}\right)$. All chemical shifts are given at ppm and using $\mathrm{CD}_{2} \mathrm{Cl}_{2}$ signals as reference $(\delta=5.30 \mathrm{ppm})$. Identification was as 


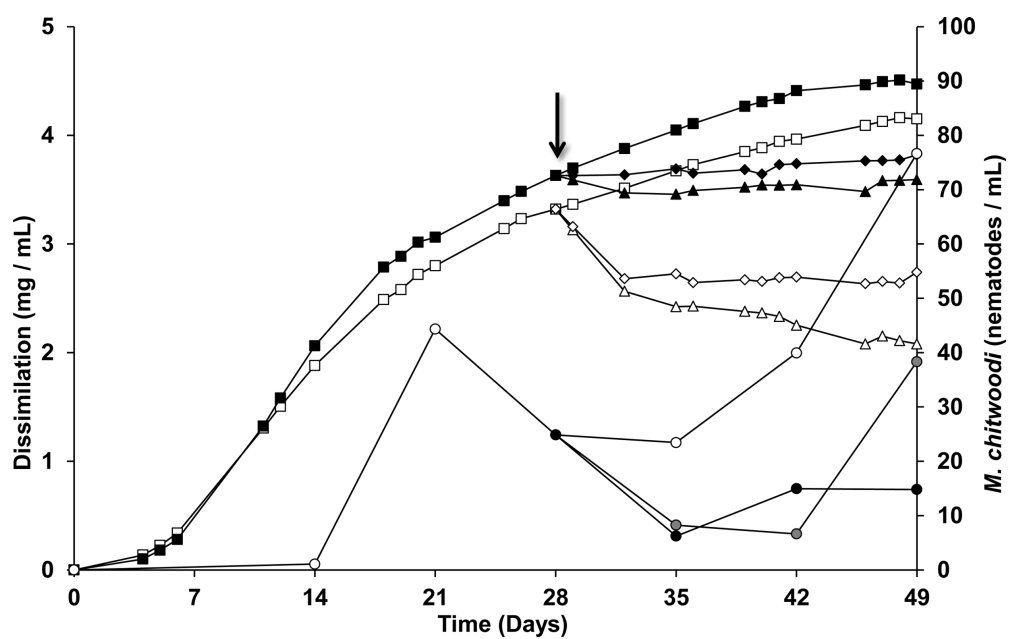

Figure 2. Dissimilation growth curves of Solanum tuberosum hairy roots (StHR, open squares) and Solanum tuberosum hairy roots with Meloidogyne chitwoodi co-cultures (StHR/CRKN, solid squares) without (StHR and StHR/CRKN) and with the addition of S. montana (open triangles and solid triangles, respectively) or $R$. graveolens essential oils (open tilted squares and solid tilted squares, respectively), at $0.5 \mu \mathrm{L} / \mathrm{mL}$ of culture medium. Number of nematodes in StHR/CRKN culture medium without (open circles) and with the addition of Satureja montana (gray solid circles) or Ruta graveolens essential oils (solid circles), at $0.5 \mu \mathrm{L} / \mathrm{mL}$ of culture medium. StHR and StHR/CRKN growth curves as in Faria et al. ${ }^{10}$ Arrow: time point of $\mathrm{EO}$ addition to the culture medium.

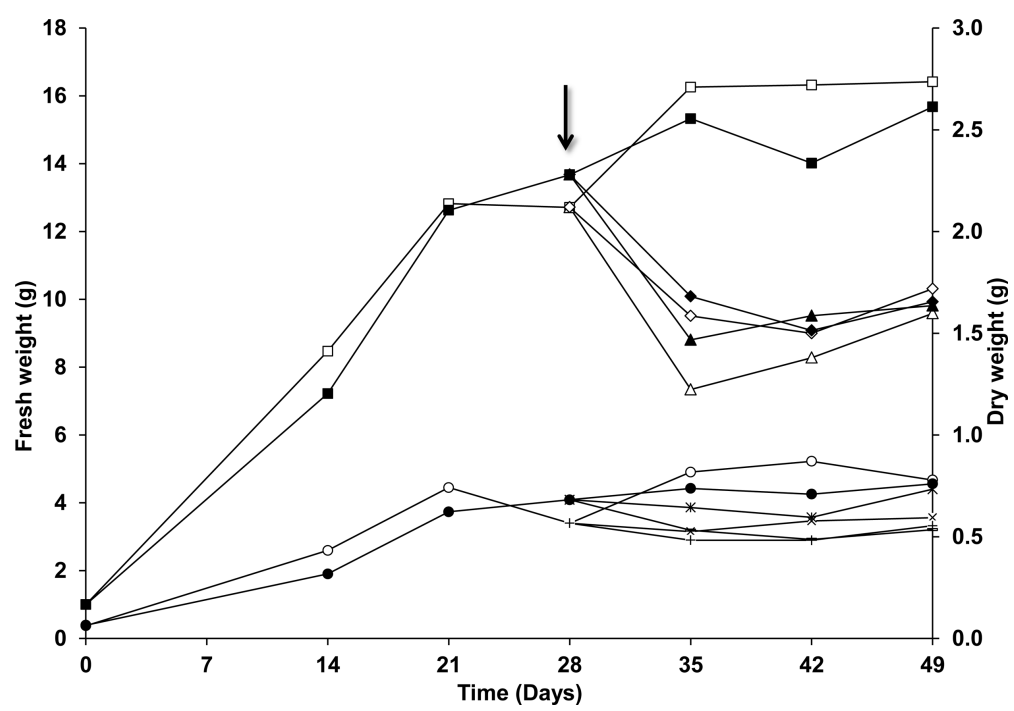

Figure 3. Fresh and dry weight growth curves of Solanum tuberosum hairy roots (StHR, open squares and open circles, respectively) and S. tuberosum hairy roots with Meloidogyne chitwoodi co-cultures (StHR/CRKN, solid squares and solid circles, respectively), without (StHR and StHR/CRKN) and with the addition of Satureja montana (fresh weight: open triangles and solid triangles, respectively; dry weight, + and - , respectively) and Ruta graveolens essential oils (fresh weight, open tilted squares and solid tilted squares, respectively; dry weight, + and - , respectively) at $0.5 \mu \mathrm{L} / \mathrm{mL} \mathrm{mL}$ of culture medium. StHR and StHR/CRKN growth curves as in Faria et al. ${ }^{10}$ Arrow: time point of EO addition to the culture medium.

follows. ${ }^{1} \mathrm{H}$ NMR $\left(400 \mathrm{MHz}, \mathrm{CD}_{2} \mathrm{Cl}_{2}\right) \delta 7.26-7.22$ (m, 2H, Ar), 7.16-7.11 (m, 3H, Ar), 3.74-3.68 (m, 1H, CH), $2.57(\mathrm{dd}, 2 \mathrm{H}, J=7.6$, $8.0 \mathrm{~Hz}), 1.58(\mathrm{~m}, 2 \mathrm{H}), 1.45(\mathrm{~m}, 8 \mathrm{H}), 1.12(3 \mathrm{H}, \mathrm{d}, 6.2 \mathrm{~Hz}) .{ }^{13} \mathrm{C} \mathrm{NMR}$ $\left(101 \mathrm{MHz}, \mathrm{CD}_{2} \mathrm{Cl}_{2}\right) \delta 143.2 \mathrm{Cq}, 128.7(2 \mathrm{CH} \mathrm{Ar}), 128.6(2 \mathrm{CH} \mathrm{Ar})$, 125.9 (CH Ar), 68.5 (CH-OH), 39.6 $\left(\mathrm{CH}_{2}\right), 36.3\left(\mathrm{CH}_{2}\right), 31.8\left(\mathrm{CH}_{2}\right)$, $29.8\left(\mathrm{CH}_{2}\right), 29.6\left(\mathrm{CH}_{2}\right), 26.0\left(\mathrm{CH}_{2}\right), 23.8\left(\mathrm{CH}_{3}\right)$. MS $(\mathrm{EI}, 70 \mathrm{eV}) \mathrm{m} /$ $z\left(\mathrm{C}_{14} \mathrm{H}_{22} \mathrm{O}\right): 206(\mathrm{M})^{+}(2), 188\left(\mathrm{M}-\mathrm{H}_{2} \mathrm{O}\right)^{+}(21), 131(10), 117(29)$, $105\left(\mathrm{C}_{8} \mathrm{H}_{9}\right)^{+}(19), 104(100), 92(34), 91\left(\mathrm{C}_{7} \mathrm{H}_{7}\right)^{+}(89), 65(10), 55$ (12), $45\left(\mathrm{C}_{2} \mathrm{H}_{5} \mathrm{O}^{+}\right)(25), 43$ (12).

\section{RESULTS AND DISCUSSION}

StHR and StHR/CRKN Growth and Volatile Profiles. $S$. tuberosum control hairy roots (StHR) showed the typical hairy root phenotype, highly branched roots with numerous root hairs (Figure 1a). ${ }^{10}$ As previously described, S. tuberosum hairy roots with $M$. chitwoodi control co-cultures (StHR/CRKN) were similar to StHR in morphology and growth (Figures 1b, 2, and 3), measured by dissimilation and fresh and dry weight methods. ${ }^{10}$ Also, CRKN in the culture medium showed the characteristic two-peak population curve (Figure 2), ${ }^{10}$ with the first peak due to first generation hatching and the second peak to $\mathrm{CRKN}$ second generation.

StHR and StHR/CRKN constitutive volatiles showed the characteristic volatile pattern, similar to that formerly reported. In total, 31 compounds were identified, of which palmitic acid (StHR 35-52\%, StHR/CRKN 24-44\%), n-pentadecanal (StHR 6-16\%, StHR/CRKN 8-22\%), linoleic acid (StHR 
Table 1. Percentage Composition Range of the Main Volatiles ( $\geq 1 \%$ ) Isolated from Solanum tuberosum Hairy Roots (StHR) and S. tuberosum HR with Meloidogyne chitwoodi Co-cultures (StHR/CRKN), during 3 Weeks after the Addition of Satureja montana EO to the Culture Medium at $0.5 \mu \mathrm{L} / \mathrm{mL}(\mathrm{StHR}+\mathrm{SmEO} \text { and StHR/CRKN }+ \text { SmEO, Respectively })^{a}$

\begin{tabular}{lccccc} 
components $(\geq 1 \%)$ & RI & StHR & StHR + SmEO & StHR/CRKN & \multicolumn{2}{c}{ StHR/CRKN + SmEO } \\
carvacrol & 1286 & $1.5-1.6$ & $75.1-85.0$ & $1.8-2.7$ & $78.3-84.1$ \\
$\beta$-caryophyllene & 1414 & nd & $1.5-3.2$ & nd & $1.5-2.6$ \\
$\beta$-bisabolene & 1500 & nd & $1.2-2.6$ & nd & t \\
$n$-pentadecanal & 1688 & $13.7-16.2$ & $1.6-3.2$ & $15.6-21.7$ & t \\
palmitic acid & 1908 & $36.7-39.5$ & $1.5-3.1$ & $32.4-38.9$ & $1.5-2.5$ \\
linoleic acid & 2101 & $6.5-7.4$ & $1.1-5.1$ & $3.7-6.5$ & nd
\end{tabular}

${ }^{a}$ For comparison purposes, StHR and StHR/CRKN volatile constitutive composition is given (StHR and StHR/CRKN), and also the percentage composition of EO evaporation/decomposition control experiments (SmEOC: EO in culture medium without StHR or StHR/CRKN), 3 weeks after EO addition. RI: In-lab calculated retention index relative to $\mathrm{C}_{12}-\mathrm{C}_{22} n$-alkanes on the DB-1 column. t: trace ( $\left.<0.05 \%\right)$. nd: not detected. [Standard deviation $<5 \%]$.

2-16\%, StHR/CRKN 4-18\%), 2-pentyl furan (StHR traces$8 \%$, StHR/CRKN 1-2\%), benzyl alcohol (StHR 3-7\%, StHR/ CRKN 3-7\%), o-guaiacol (StHR 2-4\%, StHR/CRKN 2-6\%), and $n$-hexadecanol (StHR traces-6\%, StHR/CRKN traces-2\%) were the dominant ones $(>5 \%){ }^{10}$

Satureja montana Essential Oil Bioactivity. Addition of S. montana EO at $0.5 \mu \mathrm{L} / \mathrm{mL}$ to StHR and StHR/CRKN cocultures 4 weeks following subculture (28 days, Figure 2) revealed both nematotoxic and phytotoxic effects.

CRKN population in co-cultures decreased in the first week after EO addition (35 days, Figure 2) but recovered to the end of culture time. Within the first week after EO addition, the culture medium became slightly brownish due to phenolic exudates and oxidation and the root tips dark brown when compared to those from control StHR and control StHR/ CRKN co-cultures (Figure 1c). One week after addition of $S$. montana EO, a sharp decrease in dissimilation and fresh and dry weight in both in vitro culture types was visible, comparatively to the corresponding control cultures (Figures 2 and 3). Throughout culture time, growth was not recovered to the controls growth level.

In the volatiles extracted from StHR and StHR/CRKN $S$. montana-EO added cultures, in addition to S. montana EO compounds and in vitro cultures constitutive volatiles, new volatiles were detected. S. montana EO was previously fully chemically characterized, ${ }^{15}$ showing carvacrol $(64 \%)$ and $\gamma$ terpinene $(18 \%)$ as main components. From these, only carvacrol was detected in high percentages, up to $85 \%$, in both in vitro cultures volatiles, 1 week after EO addition. Although with a tendency to decrease, carvacrol percentage remained high even 3 weeks after EO addition (StHR 75\%, StHR/CRKN $78 \%$ ) (Table 1).

$\gamma$-Terpinene was only detected in trace amounts, which can be partly attributed to volatilization, as this was also detected in control experiments of EO evaporation and decomposition. However, substrate hydroxylation, glycosylation, oxidoreduction, hydrogenation, hydrolysis, methylation, acetylation, isomerization, and esterification are some biotransformation reactions commonly found on plant in vitro cultures. ${ }^{17-21}$ For this reason, the biotransformation of $\gamma$-terpinene into nonvolatile glycosylated compounds can also partly explain the difference between $\gamma$-terpinene percentage in S. montana EO and $S$. montana EO added cultures. Seven new compounds were detected (all of which $<0.3 \%$ ) in the volatiles extracted from StHR and StHR/CRKN S. montana-EO added cultures, carvone, thymoquinone, tridecanal, and four, as yet unidentified, compounds. Oxidation, isomerization, and/or reduction

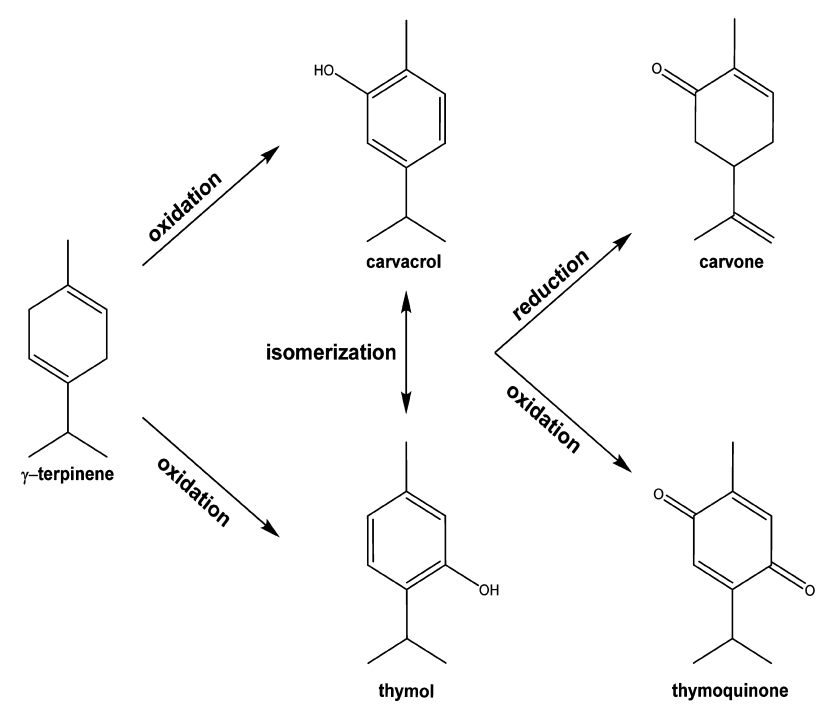

Figure 4. Putative biotransformation reactions of Satureja montana EO dominant compounds, carvacrol and $\gamma$-terpinene, by Solanum tuberosum hairy roots, or $S$. tuberosum HR with Meloidogyne chitwoodi co-cultures.

(Figure 4) are biotransformation reactions that can explain the conversion of S. montana EO dominant compounds, carvacrol and $\gamma$-terpinene, into the new compounds, carvone and thymoquinone, present in the volatiles in trace amounts.

S. montana EOs, commonly dominated by carvacrol, $\gamma$ terpinene, and/or $p$-cymene, are known to possess high phytotoxic bioactivities. Angelini et al. ${ }^{22}$ reported complete in vitro germination inhibition of three weeds and three crops seeds subjected to $S$. montana $\mathrm{EO}$ at $0.5 \mathrm{mg} / \mathrm{mL}$. In an attempt to pinpoint the main phytotoxic compound, further testing was performed with carvacrol, the main component (57\%), and again similar inhibition activities were obtained.

For testing phytotoxic synergic activities of monoterpenes, carvacrol was assayed against Lactuca sativa. ${ }^{23}$ Alone or in synergic combination with $\gamma$-terpinene or $p$-cymene, high activities were obtained in the inhibition of germination and seedling elongation. Carvacrol-rich EOs showed high sprouting inhibition on stored S. tuberosum tubers. ${ }^{24,25}$ Kordali et al. ${ }^{26}$ analyzed the phytotoxic potential of Origanum acutidens EO, and its three main components, carvacrol, thymol, and $p$ cymene, on seeds of Amaranthus retroflexus, Chenopodium album, and Rumex crispus weeds. This study showed that the EO, carvacrol, or thymol completely inhibited in vitro seed 
Table 2. Percentage Composition Range of the Main Volatiles ( $\geq 1 \%$ ) Isolated from Solanum tuberosum Hairy Roots (StHR) and S. tuberosum HR with Meloidogyne chitwoodi Co-cultures (StHR/CRKN), during 3 Weeks after the Addition of Ruta graveolens EO to the Culture Medium at $0.5 \mu \mathrm{L} / \mathrm{mL}(\mathrm{StHR}+\mathrm{RgEO} \text { and StHR/CRKN }+ \text { RgEO, respectively })^{a}$

\begin{tabular}{|c|c|c|c|c|c|c|}
\hline components $(\geq 1 \%)$ & RI & StHR & $\mathrm{StHR}+\mathrm{RgEO}$ & StHR/CRKN & StHR/CRKN + RgEO & $\mathrm{RgEOC}$ \\
\hline 2-undecanone & 1275 & nd & $4.7-15.6$ & nd & $4.6-16.5$ & 2.3 \\
\hline 2-undecanol & 1288 & nd & $1.3-6.1$ & nd & $1.5-5.7$ & 1.5 \\
\hline 2-dodecanone & 1389 & nd & $0.3-2.8$ & nd & $0.3-3.6$ & 0.2 \\
\hline 2-tridecanone & 1479 & nd & $0.6-5.5$ & nd & $0.5-5.8$ & 0.4 \\
\hline$n$-tridecanol & 1565 & nd & $0.3-1.2$ & nd & $0.3-1.2$ & nd \\
\hline$n$-tetradecanal & 1596 & $1.6-2.1$ & $0.9-1.6$ & $2.4-3.6$ & $1.0-1.7$ & nd \\
\hline 8-phenyl-2-octanone & 1626 & nd & $54.3-66.9$ & nd & $52.4-68.1$ & 75.1 \\
\hline 8-phenyl-2-octanol & 1640 & nd & $2.3-4.3$ & nd & $2.3-4.8$ & 1.1 \\
\hline$n$-tetradecanol & 1659 & nd & $1.1-4.3$ & nd & $1.0-4.7$ & nd \\
\hline$n$-pentadecanal & 1688 & $13.7-16.2$ & $2.2-3.5$ & $15.6-21.7$ & $2.7-4.0$ & nd \\
\hline UI D $\mathrm{Rg}^{b}$ & 1775 & nd & $0.6-1.0$ & nd & $0.5-0.9$ & 7.0 \\
\hline UI $E \mathrm{Rg}^{b}$ & 1784 & nd & $0.8-0.9$ & nd & $0.8-1.0$ & 0.8 \\
\hline palmitic acid & 1908 & $36.7-39.5$ & $0.8-2.0$ & $32.4-38.9$ & $0.5-1.3$ & nd \\
\hline
\end{tabular}

${ }^{a}$ For comparison purposes, StHR and StHR/CRKN volatile constitutive compositions are given (StHR and StHR/CRKN) and also the percentage composition of EO evaporation/decomposition control experiments (RgEOC: EO in culture medium without StHR or StHR/CRKN), 3 weeks after EO addition. RI: In-lab calculated retention index relative to $C_{12}-C_{22} n$-alkanes on the DB- 1 column. $t$ : trace ( $\left.<0.05 \%\right)$. nd: not detected. ${ }^{b}$ Unidentified compounds detected in $R$. graveolens essential oil. [Standard deviation <5\%].

germination and seedling growth, and their activity was higher than that of commercial herbicide, 2,4-D isooctyl ester. Also Azirak and Karaman ${ }^{27}$ found high phytotoxic activities for carvacrol and thymol-rich EOs and respective synthetic chemicals. These EOs and compounds inhibited in vitro germination of six weeds found in field and horticultural crops. When added to Pinus pinaster shoot cultures and P. pinaster with Bursaphelenchus xylophilus in vitro co-cultures, S. montana EO also demonstrated a high phytotoxic activity, inducing shoot chlorosis and wilting while maintaining nematotoxic activity. ${ }^{13}$

Ruta graveolens Essential Oil Bioactivity. Growth of StHR and StHR/CRKN co-cultures was completely inhibited after $R$. graveolens $\mathrm{EO}$ addition, the root tips turning dark brown (Figure 1d). Similarly to what happened after the addition of $S$. montana EO, $R$. graveolens EO inhibited StHR/CRKN nematode population. One week after $R$ graveolens EO addition, CRKN population decreased $<10 \mathrm{CRKN} / \mathrm{mL}$ culture medium and this inhibitory effect was maintained throughout (Figure 2).

$R$ graveolens EO compounds, in vitro cultures constitutive compounds and also new ones, were found in $R$ graveolens EO added in vitro cultures isolated volatiles. Previously identified $^{13,15}$ main $R$. graveolens EO compounds, 2-undecanone (91\%) and 8-phenyl-2-octanone (7\%), were detected on StHR and StHR/CRKN co-cultures EO-added volatiles. The ketone, 8-phenyl-2-octanone dominated StHR (54-67\%), and StHR/ CRKN co-cultures $(52-68 \%)$ volatiles, $R$. graveolens EO main compound, 2-undecanone, showed lower relative amounts than 8-phenyl-2-octanone and decreased throughout experimental time in StHR (16-5\%) and StHR/CRKN (17-5\%) cocultures (Table 2).

Twenty-nine new compounds were detected after $R$. graveolens EO addition to StHR cultures and StHR/CRKN co-cultures: $n$-decanol, $n$-dodecanol, elemol, $\beta$-eudesmol, 2,4heptadienal, $n$-hexadecanal, 5-methylene-2,3,4,4-tetramethylcyclopent-2-enone, 1-octen-3-ol, 6-phenyl-n-hexanol, 6-phenyl-2hexanone, 2-trans-4-cis-decadienal, trans-nerolidol, trans-2nonen-1-al, $n$-tetradecane, $n$-tetradecanol, tetradecanol allyl ether, 1 -tetradecene, $n$-tridecanal, $n$-tridecane, $n$-tridecanol, $n$ - undecanol, and eight unidentified compounds. Compounds present $>1 \%$ are listed in Table 2 .

The phytotoxic properties of $R$. graveolens EO and some EO compounds were reported by de Feo et al. ${ }^{28}$ on Raphanus sativus seeds. In vitro germination and seedling radicle growth were inhibited by $R$. graveolens $\mathrm{EO}$ and some minor constituents, but not by the major components, 2-undecanone or 2-nonanone, when tested separately. The addition of $R$. graveolens EO to Pinus pinaster with Bursaphelenchus xylophilus in vitro co-cultures showed no visible phytotoxic effects in shoot aspect and volatiles composition. ${ }^{13}$ Methyl ketones, particularly 2-undecanone, are currently used as insect and animal repellents in households, paths, patios, solid waste containers, and on ornamental plants. ${ }^{29}$ Their activity against various Solanum spp. pests has been tested by Antonious et al. ${ }^{30}$ Isolated from crude extracts of resistant wild tomato plants, Lycopersicon hirsutum, it has shown to be promising as herbicidal for weed control. ${ }^{31}$

Noma and Asakawa ${ }^{32}$ analyzed the biotransformation capacity of the alga Euglena gracilis $\mathrm{Z}$ strain by feeding a series of methyl ketones. The authors concluded that all compounds were reduced to the corresponding alcohols with a certain hierarchy of preference that was related to the length of the side chain. The longer side chain of aliphatic methyl ketones increased the reactivity for the reduction of the carbonyl group. In the present study, EO methyl ketones were also reduced to their corresponding alcohols, having been detected in the new induced compounds, main compounds 2-undecanone and 8phenyl-2-octanone were reduced to 2-undecanol and 8-phenyl2-octanol, as identified through NMR and GC-MS. Reduction reactions may constitute a detoxification response to the introduction of bioactive ketones.

Using Solanum tuberosum hairy roots with Meloidogyne chitwoodi co-cultures, the effect of adding nematotoxic $S$. montana and R. graveolens EOs was evaluated in a host-parasite system. Both EOs revealed phytotoxicity toward the StHR and $\mathrm{StHR} / \mathrm{CRKN}$ co-cultures. In spite of this, $R$. graveolens $\mathrm{EO}$ was able to control parasite growth for a longer period even though some major EO compounds may have been biotransformed. In vitro co-cultures used as biotechnological models can 
contribute to a more expeditious screening procedure and establishment of the effectiveness of nematotoxic EOs by allowing a preview of how the plant host reacts to nematotoxics. Nevertheless, it must be noted that the observed nematoxicity in a co-culture system cannot be inputted exclusively to the EO, as the host upon which the nematode feeds can also biotransform the EO in such a way that it changes its composition and thus the EO bioactivity. Moreover, the pronounced phytotoxicity of these EOs on potato HR alerts to their cautionary use as nematicidals. This knowledge may help in designing further assays on in vivo root-knot diseased plants to determine its activity under field conditions.

\section{AUTHOR INFORMATION}

\section{Corresponding Author}

*Phone: +351217500257. Fax: 00351217500048. E-mail: acsf@fc.ul.pt.

\section{Funding}

M. Mota was partially supported by Fundação para a Ciência e a Tecnologia (FCT) under Pest-C/AGR/UI0115/2011 and PEst-OE/AGR/UI0115/2014. Jorge Faria is grateful to FCT for the $\mathrm{PhD}$ grant SFRH/BD/43738/2008. This study was partially funded by FCT, under Pest-OE/EQB/LA0023/2011, UID/AMB/50017/2013, FEDER PT2020-Compete 2020, and research contract PTDC/AGR-CFL/117026/2010.

\section{Notes}

The authors declare no competing financial interest.

\section{ABBREVIATIONS USED}

CRKN, Columbia root-knot nematode; EO, essential oil; HR, hairy roots; Rg, Ruta graveolens essential oil; RI, retention index; SH, Schenk and Hildebrandt culture medium; StHR, Solanum tuberosum hairy roots; StHR/CRKN, Solanum tuberosum hairy roots with Columbia root-knot nematode cocultures; $\mathrm{t}$, trace

\section{REFERENCES}

(1) Jones, J. T.; Haegeman, A.; Danchin, E. G. J.; Gaur, H. S.; Helder, J.; Jones, M. G. K.; Kikuchi, T.; Manzanilla-Lopéz, R.; Palomares-Rius, J. E.; Wesemael, W. M. L.; Perry, R. N. Top 10 plant-parasitic nematodes in molecular plant pathology. Mol. Plant Pathol. 2013, 14, 946-961.

(2) Chitwood, D. J. Nematicides. In Encyclopedia of Agrochemicals; Plimmer, J. R., Ed.; John Wiley \& Sons: New York, 2003; Vol. 3, pp 1104-1115.

(3) Palomares-Rius, J. E.; Oliveira, C. M. G.; Blok, V. C. Plant parasitic nematodes of potato. In The Potato. Botany, Production and Uses.; Navarre, R., Pavek, M. J., Eds.; CAB International; United States Department of Agriculture; Wallingford, Oxfordshire, UK; Boston, MA, 2014; pp 148-166.

(4) Batish, D. R.; Singh, H. P.; Kohli, R. K.; Kaur, S. Eucalyptus essential oil as a natural pesticide. For. Ecol. Manage. 2008, 256, 21662174.

(5) Oka, Y.; Nacar, S.; Putievsky, E.; Ravid, U.; Yaniv, Z.; Spiegel, Y. Nematicidal activity of essential oils and their components against the root-knot nematode. Phytopathology 2000, 90, 710-715.

(6) Pérez, M. P.; Navas-Cortés, J. A.; Pascual-Villalobos, M. J.; Castillo, P. Nematicidal activity of essential oils and organic amendments from Asteraceae against root-knot nematodes. Plant Pathol. 2003, 52, 395-401.

(7) Ibrahim, S. K.; Traboulsi, A. F.; El-Haj, S. Effect of essential oils and plant extracts on hatching, migration and mortality of Meloidogyne incognita. Phytopathol. Mediterr. 2006, 45, 238-246.

(8) Meyer, S. L. F.; Lakshman, D. K.; Zasada, I. A.; Vinyard, B. T.; Chitwood, D. J. Dose-response effects of clove oil from Syzygium aromaticum on the root-knot nematode Meloidogyne incognita. Pest Manage. Sci. 2008, 64, 223-229.

(9) Andrés, M. F.; González-Coloma, A.; Sanz, J.; Burillo, J.; Sainz, P. Nematicidal activity of essential oils: a review. Phytochem. Rev. 2012, 11, 371-390.

(10) Faria, J. M. S.; Sena, I.; Maleita, C. M.; Vieira da Silva, I.; Ascensão, L.; Abrantes, I.; Bennett, R. N.; Mota, M.; Figueiredo, A. C. In vitro co-culture of Solanum tuberosum hairy roots with Meloidogyne chitwoodi: structure, growth and production of volatiles. Plant Cell, Tissue Organ Cult. 2014, 118, 519-530.

(11) Faria, J. M. S.; Sena, I.; Vieira da Silva, I.; Ribeiro, B.; Barbosa, P.; Ascensão, L.; Bennett, R. N.; Mota, M.; Figueiredo, A. C. In vitro co-cultures of Pinus pinaster with Bursaphelenchus xylophilus: a biotechnological approach to study pine wilt disease. Planta 2015, 241, 1325-1336.

(12) Faria, J. M. S.; Sena, I.; Ribeiro, B.; Rodrigues, A. M.; Maleita, C. M.; Abrantes, I.; Bennett, R. N.; Mota, M.; Figueiredo, A. C. First report on Meloidogyne chitwoodi hatching inhibition activity of essential oils and essential oils fractions. J. Pest Sci. 2016, 89, 207-217.

(13) Faria, J. M. S.; Sena, I.; Moiteiro, C.; Bennett, R. N.; Mota, M.; Figueiredo, A. C. Nematotoxic and phytotoxic activity of Satureja montana and Ruta graveolens essential oils on Pinus pinaster shoot cultures and P. pinaster with Bursaphelenchus xylophilus in vitro co cultures. Ind. Crops Prod. 2015, 77, 59-65.

(14) Schenk, U. R; Hildebrandt, A. C. Medium and techniques for induction and growth of monocotyledonous and dicotyledonous plant cell cultures. Can. J. Bot. 1972, 50, 199-204.

(15) Faria, J. M. S.; Barbosa, P.; Bennett, R. N.; Mota, M.; Figueiredo, A. C. Bioactivity against Bursaphelenchus xylophilus: nematotoxics from essential oils, essential oils fractions and decoction waters. Phytochemistry 2013, 94, 220-228.

(16) Still, W. C.; Kahn, M.; Mitra, A. Rapid chromatographic technique for preparative separations with moderate resolution. J. Org. Chem. 1978, 43, 2923-2925.

(17) Figueiredo, A. C.; Almendra, M. J.; Barroso, J. G.; Scheffer, J. J. C. Biotransformation of monoterpenes and sesquiterpenes by cell suspension cultures of Achillea millefolium L. ssp. millefolium. Biotechnol. Lett. 1996, 18, 863-868.

(18) Giri, A.; Dhingra, V.; Giri, C. C.; Singh, A.; Ward, O. P.; Narasu, M. P. Biotransformations using plant cells, organ cultures and enzyme systems: current trends and future prospects. Biotechnol. Adv. 2001, 19, $175-199$.

(19) Faria, J. M. S.; Nunes, I. S.; Figueiredo, A. C.; Pedro, L. G.; Trindade, H.; Barroso, J. G. Biotransformation of menthol and geraniol by hairy root cultures of Anethum graveolens: effect on growth and volatile components. Biotechnol. Lett. 2009, 31, 897-903.

(20) Nunes, I. S.; Faria, J. M. S.; Figueiredo, A. C.; Pedro, L. G.; Trindade, H.; Barroso, J. G. Menthol and geraniol biotransformation and glycosylation capacity of Levisticum officinale hairy roots. Planta Med. 2009, 75, 387-391.

(21) Banerjee, S.; Singh, S.; Rahman, L. U. Biotransformation studies using hairy root cultures - A review. Biotechnol. Adv. 2012, 30, 461468.

(22) Angelini, L. G.; Carpanese, G.; Cioni, P. L.; Morelli, I.; Macchia, M.; Flamini, G. Essential oils from mediterranean Lamiaceae as weed germination inhibitors. J. Agric. Food Chem. 2003, 51, 6158-6164.

(23) Vokou, D.; Douvli, P.; Blionis, G. J.; Halley, J. M. Effects of monoterpenoids, acting alone or in pairs, on seed germination and subsequent seedling growth. J. Chem. Ecol. 2003, 29, 2281-2301.

(24) Vokou, D.; Vareltzidou, S.; Katinakis, P. Effects of aromatic plants on potato storage: sprout suppression and antimicrobial activity. Agric., Ecosyst. Environ. 1993, 47, 223-235.

(25) Baydar, H.; Karadoğan, T. The effects of volatile oils on in vitro potato sprout growth. Potato Res. 2003, 46, 1-8.

(26) Kordali, S.; Cakir, A.; Ozer, H.; Cakmakci, R.; Kesdek, M.; Mete, E. Antifungal, phytotoxic and insecticidal properties of essential oil isolated from Turkish Origanum acutidens and its three components, carvacrol, thymol and p-cymene. Bioresour. Technol. 2008, 99, 87888795. 
(27) Azirak, S.; Karaman, S. Allelopathic effect of some essential oils and components on germination of weed species. Acta Agric. Scand., Sect. B 2008, 58, 88-92.

(28) de Feo, V.; de Simone, F.; Senatore, F. Potential allelochemicals from the essential oil of Ruta graveolens. Phytochemistry 2002, 61, 573578.

(29) Methyl Nonyl Ketone. In R.E.D. Facts; Office of Prevention, Pesticides and Toxic Substances, U.S. Environmental Protection Agency: Washington, DC, 1995; https://archive.epa.gov/pesticides/ reregistration/web/pdf/3094fact.pdf (accessed July 19, 2016).

(30) Antonious, G. F.; Kamminga, K.; Snyder, J. C. Wild tomato leaf extracts for spider mite and cowpea aphid control. J. Environ. Sci. Health, Part B 2014, 49, 527-531.

(31) Bradow, J. M.; Connick, W. J. Volatile methyl ketone seedgermination inhibitors from Amaranthus palmeri S. Wats. Residues. J. Chem. Ecol. 1988, 14, 1617-631.

(32) Noma, Y.; Asakawa, Y. Euglena gracilis Z Biotransformation of Terpenoids and Related Compounds. In Biotechnology in Agriculture and Forestry: Medicinal and Aromatic Plants; Bajaj, Y. P. S., Ed.; Springer: New York, 1998; Vol. 10, pp 194-237. 